Research Article

\title{
Strong Convergence of a Monotone Projection Algorithm in a Banach Space
}

\author{
Songtao Lv \\ School of Mathematics and Information Science, Shangqiu Normal University, Shangqiu 476000, China \\ Correspondence should be addressed to Songtao Lv; sqlvst@yeah.net
}

Received 1 September 2013; Accepted 20 October 2013

Academic Editors: K. Ammari and K. Zhu

Copyright (C) 2013 Songtao Lv. This is an open access article distributed under the Creative Commons Attribution License, which permits unrestricted use, distribution, and reproduction in any medium, provided the original work is properly cited.

In this paper, a common solution problem is investigated based on a Bregman projection. Strong convergence of the monotone projection algorithm for monotone operators and bifunctions is obtained in a reflexive Banach space.

\section{Introduction}

Fixed point theory as an important branch of nonlinear analysis theory has been applied in the study of nonlinear phenomena. Lots of problems arising in economics, engineering, and physics can be studied based on fixed point techniques; see [1-21] and the references therein. Many wellknown problems can be studied by using algorithms which are iterative in their nature. As an example, in computer tomography with limited data, each piece of information implies the existence of a convex set $C_{m}$ in which the required solution lies. The problem of finding a point in the intersection $\cap_{m=1}^{N} C_{m}$, where $N \geq 1$ is some positive integer, is then of crucial interest, and it cannot be usually solved directly. Therefore, an iterative algorithm must be used to approximate such point. The well-known convex feasibility problem which captures applications in various disciplines such as image restoration and radiation therapy treatment planning is to find a point in the intersection of common fixed point sets of a family of nonlinear mappings see, for example, [22-28].

Interest in monotone operators stems mainly from their firm connection with equations of evolution which is an important class of nonlinear operators. It is known that many physically significant problems can be modelled by initial value problems of the form:

$$
x^{\prime}(t)+A x(t)=0, \quad x(0)=x_{0},
$$

where $A$ is an monotone operator in an appropriate Banach space. Typical examples, where such evolution equations occur, can be found in the heat, wave, or Schrödinger's equations. If $x(t)$ is dependent on $t$, then (1) is reduced to

$$
A u=0,
$$

whose solutions correspond to the equilibrium points of the system (1). Consequently, considerable research efforts have been devoted, especially within the past 40 years or so, to methods for finding approximate solutions (when they exist) of (2). An early fundamental result in the theory of monotone operators, due to Browder [29], states that the initial value problem (1) is solvable if $A$ is locally Lipschitz and accretive on $E$.

The Krasnoselskii-Mann iterative algorithm is efficient for treating zero points of monotone operators. However, it is weak convergence only. In many disciplines, including economics, image recovery, and control theory, problems arise in infinite dimension spaces. In such problems, strong convergence (norm convergence) is often much more desirable than weak convergence for it translates the physically tangible property that the energy $\left\|x_{n}-x\right\|$ of the error between the iterate $x_{n}$ and the solution $x$ eventually becomes arbitrarily small. The importance of strong convergence is also underlined, where a convex function $f$ is minimized via the proximal-point algorithm; it is shown that the rate of convergence of the value sequence $\left\{f\left(x_{n}\right)\right\}$ is better when $\left\{x_{n}\right\}$ converges strongly than when it converges weakly. Such properties have a direct impact when the process is executed directly in the underlying infinite dimensional space. Projection methods which were first introduced by Haugazeau [30] 
have been considered for treating zero points of monotone operators. The advantage of projection methods is that strong convergence of iterative sequences can be guaranteed without any compact assumptions.

In this paper, we study common zero points of a family of maximal monotone operators and common solutions of a system of equilibrium problems based on the Bregman projection. Strong convergence of the monotone projection algorithm is obtained in a reflexive Banach space.

\section{Preliminaries}

Let $E$ be a real Banach space, $E^{*}$ the dual space of $E$, and $C$ a nonempty subset of $E$. Let $f$ be a bifunction from $C \times C$ to $\mathbb{R}$, where $\mathbb{R}$ denotes the set of real numbers. Recall the following equilibrium problem. Find $\bar{x} \in C$ such that

$$
f(\bar{x} y) \geq 0, \quad \forall y \in C .
$$

We use $\operatorname{EP}(f)$ to denote the solution set of the equilibrium problem (3). That is,

$$
\mathrm{EP}(f)=\{p \in C: f(p, y) \geq 0, \forall y \in C\} .
$$

Given a mapping $B: C \rightarrow E^{*}$, let

$$
f(x, y)=\langle B x, y-x\rangle, \quad \forall x, y \in C .
$$

Then, $\bar{x} \in \operatorname{EP}(f)$ if and only if $\bar{x}$ is a solution of the following variational inequality. Find $\bar{x}$ such that

$$
\langle B \bar{x} y-\bar{x}\rangle \geq 0, \quad \forall y \in C .
$$

In order to study the solution of the equilibrium problem (3), we assume that $f$ satisfies the following conditions:

(A1) $f(x, x)=0$, for all $x \in C$;

(A2) $f$ is monotone; that is, $f(x, y)+f(y, x) \leq 0$, for all $x, y \in C$

$$
\limsup _{t \downarrow 0} f(t z+(1-t) x, y) \leq f(x, y), \quad \forall x, y, z \in C ;
$$

(A4) for each $x \in C, y \mapsto f(x, y)$ is convex and weakly lower semicontinuous.

For any convex function $g: E \rightarrow(-\infty,+\infty]$, we denote the domain of $g$ by $\operatorname{Dom}(g)=\{x \in E: g(x)<\infty\}$. For any $x \in$ int $\operatorname{Dom}(g)$ and $y \in E$, we denote by $g^{0}(x, y)$ the righthand derivative of $g$ at $x$ in the direction $y$; that is,

$$
g^{0}(x, y)=\lim _{t \rightarrow 0^{+}} \frac{g(x+t y)-g(x)}{t} .
$$

The function $g$ is said to be Gâteaux differentiable at $x$ if $\lim _{t \rightarrow 0^{+}}((g(x+t y)-g(x)) / t)$ exists for any $y$. In this case, $g^{0}(x, y)$ coincides with $\nabla g(x)$, the value of the gradient $\nabla g$ of $g$ at $x$. The function $g$ is said to be Gâteaux differentiable if it is Gâteaux differentiable everywhere. The function $g$ is said to be Fréchet differentiable at $x$ if the limit is attained uniformly in $\|y\|=1$. The function $g$ is said to be Fréchet differentiable if it is Fréchet differentiable everywhere. It is well known that if a continuous convex function $g: E \rightarrow \mathbb{R}$ is Gâteaux differentiable, then $\nabla g$ is norm-to-weak ${ }^{*}$ continuous. If $g: E \rightarrow \mathbb{R}$ is Fréchet differentiable, then $\nabla g$ is norm-to-norm continuous; for more details, see [31] and the references therein; for more details, see [32] and the references therein. The function $g$ is said to be strongly coercive [32] if $\lim _{\|x\| \rightarrow \infty}(f(x) /\|x\|)=\infty$. It is said to be bounded on bounded subsets of $E$ if $g(B)$ is bounded for each bounded subset $B$ of $E$. Let $E$ be a reflexive Banach space. For any proper, lower semicontinuous, and convex function: $g: E \rightarrow$ $(-\infty,+\infty]$, the conjugate function $g^{*}$ of $g$ is defined by

$$
g^{*}\left(x^{*}\right)=\sup _{x \in E}\left\{\left\langle x, x^{*}\right\rangle-g(x)\right\}, \quad \forall x^{*} \in E^{*} .
$$

It is well known that $g(x)+g^{*}\left(x^{*}\right) \geq\left\langle x, x^{*}\right\rangle$ for all $\left(x, x^{*}\right) \epsilon$ $E \times E^{*}$. It is also known that $\left(x, x^{*}\right) \in \partial g$, where $\partial g$ is the subdifferential of $g$, is equivalent to

$$
g(x)+g^{*}\left(x^{*}\right)=\left\langle x, x^{*}\right\rangle .
$$

If $g$ is a proper, lower semicontinuous, and convex function, then $g^{*}$ is a proper, weak ${ }^{*}$ lower semicontinuous, and convex function.

Next, we recall some facts about Bregman distance. Let $g: E \rightarrow \mathbb{R}$ be a convex and Gâteaux differentiable function. Then, Bregman distance corresponding to $g$ is the function $D_{g}: E \times E \rightarrow \mathbb{R}$ defined by

$$
D_{g}(x, y)=g(x)-g(y)-\langle x-y, \nabla g(y)\rangle, \quad \forall x, y \in E .
$$

It is clear that $D_{g}(x, y) \geq 0$ for all $x, y \in E$. If $E$ is smooth and $g(x)=\|x\|^{2}$ for all $x \in E$, we obtain that $\nabla g(x)=2 J x$, where $J$ is the generalized duality mapping. If $C$ is a nonempty, closed and convex subset of a reflexive Banach space $E$ and $g$ is a strongly coercive Bregman function, then for each $x \in E$, there exists a unique $x_{0} \in C$ such that $D_{g}\left(x_{0}, x\right)=$ $\min _{y \in C} D_{g}(y, x)$. Bregman projection $\operatorname{Proj}_{C}^{g}$ from $E$ onto $C$ is defined by $\operatorname{Proj}_{C}^{g} x=x_{0}$ for all $x \in E$. It is also well known [32] that $\operatorname{Proj}_{C}^{g}$ has the following property:

$$
\begin{array}{r}
D_{g}\left(y, \operatorname{Proj}_{C}^{g} x\right)+D_{g}\left(\operatorname{Proj}_{C}^{g} x, x\right) \leq D_{g}(y, x), \\
\forall y \in C, x \in E .
\end{array}
$$

Let $B_{r}:=\{z \in E:\|z\|<r\}$. A function $g: E \rightarrow \mathbb{R}$ is said to be uniformly convex on bounded subsets of $E$ if $f_{r}(t)>0$ for all $r, t>0$, where $f_{r}:[0, \infty] \rightarrow \infty[0, \infty]$ is defined by

$$
\begin{aligned}
f_{r}(t)=\inf _{x, y \in B_{r},\|x-y\|=t, \alpha \in(0,1)}( & (\alpha g(x)+(1-\alpha) g(y) \\
& -g(\alpha x+(1-\alpha) y)) \\
& \left.\times(\alpha(1-\alpha))^{-1}\right), \quad \forall t \geq 0 .
\end{aligned}
$$


The function $f_{r}$ is called the gauge of the uniform convexity of $g$. If $g: E \rightarrow \mathbb{R}$ is a convex function which is uniformly convex on bounded subsets, then

$$
\begin{aligned}
g(\alpha x+(1-\alpha) y) \leq & \alpha g(x)+(1-\alpha) g(y) \\
& -\alpha(1-\alpha) f_{r}(\|x-y\|)
\end{aligned}
$$

for all $x, y \in B_{r}$ and $\alpha \in(0,1)$, where $f_{r}$ is the gauge of the uniform convexity of $g$.

Let $T: C \rightarrow C$ be a mapping. In this paper, we use $F(T)$ to denote the fixed point set of $T$. $T$ is said to be closed; if for any sequence $\left\{x_{n}\right\} \subset C$ such that $\lim _{n \rightarrow \infty} x_{n}=x_{0}$ and $\lim _{n \rightarrow \infty} T x_{n}=y_{0}$, then $T x_{0}=y_{0}$.

Let $g: E \rightarrow \mathbb{R}$ be a proper, lower semicontinuous and convex function. Recall that $T: C \rightarrow C$ is said to be Bregman quasi-nonexpansive, if and only if

$$
\begin{array}{r}
F(T) \neq \emptyset, \quad D_{g}(p, T x) \leq D_{g}(p, x), \\
\forall x \in C, \quad p \in F(T) .
\end{array}
$$

A point $p$ in $C$ is said to be an asymptotic fixed point of $T$ if and only if $C$ contains a sequence $\left\{x_{n}\right\}$, which converges weakly to $p$ such that $\lim _{n \rightarrow \infty}\left\|x_{n}-T x_{n}\right\|=0$. The set of asymptotic fixed points of $T$ will be denoted by $\widetilde{F}(T)$. $T$ is said to be Bregman relatively nonexpansive if and only if $\widetilde{F}(T)=$ $F(T) \neq \emptyset$ and $D_{g}(p, T x) \leq D_{g}(p, x)$ for all $x \in C$ and $p \in$ $F(T)$.

Let $A$ be a multivalued operator from $E$ to $E^{*}$ with domain $\operatorname{Dom}(A)=\{z \in E: A z \neq \emptyset\}$ and range $\operatorname{Ran}(A)=$ $\cup\{A z: z \in \operatorname{Dom}(A)\}$. An operator $A$ is said to be monotone if and only if $\left\langle x_{1}-x_{2}, y_{1}-y_{2}\right\rangle \geq 0$ for each $x_{i} \in \operatorname{Dom}(A)$ and $y_{i} \in A x_{i}, i=1,2$. A monotone operator $A$ is said to be maximal if its graph $\operatorname{Grap}(A)=\{(x, y): y \in A x\}$ is not properly contained in the graph of any other monotone operator. We know that if $A$ is a maximal monotone operator, then $A^{-1}(0)$ is closed and convex.

Let $E$ be a reflexive, strictly convex, and smooth Banach space, and let $A$ be a maximal monotone operator from $E$ to $E^{*}$. From Rockafellar [33], we find that $s>0$ and $x \in E$; there exists a unique $x_{s} \in D(A)$ such that $\nabla g x \in \nabla g x_{s}+s A x_{s}$. If $J_{s} x=x_{s}$, then we can define a single-valued mapping $J_{s}$ : $E \rightarrow \operatorname{Dom}(A)$ by $J_{s}=(\nabla g+s A)^{-1} \nabla g$ and such a $J_{s}$ is called the resolvent of $A$. We know that $J_{s}$ is closed and $A^{-1}(0)=$ $F\left(J_{s}\right)$ for all $s>0$. From [34], we know that $J_{s}: E \rightarrow \operatorname{Dom}(A)$ is a Bregman quasi-nonexpansive.

In this paper, we study a common solution problem based on Bregman projections. Strong convergence of the monotone projection algorithm for monotone operators and bifunctions is obtained in a reflexive Banach space.

In order to introduce our main results, we also need the following lemmas.

Lemma 1 (see [34]). Let $E$ be a Banach space and let $g: E \rightarrow$ $\mathbb{R}$ be a Gâteaux differentiable function which is uniformly convex on bounded subsets of E. Let $\left\{x_{n}\right\}$ and $\left\{y_{n}\right\}$ be bounded sequences in $E$. Then,

$$
\lim _{n \rightarrow \infty} D_{g}\left(x_{n}, y_{n}\right)=0 \Longleftrightarrow \lim _{n \rightarrow \infty}\left\|x_{n}-y_{n}\right\|=0 .
$$

Lemma 2 (see [35]). Let $E$ be a reflexive Banach space and let $g: E \rightarrow \mathbb{R}$ be a convex, continuous, strongly coercive, and Gâteaux differentiable function which is bounded on bounded subsets and uniformly convex on bounded subsets of E. Let $C$ be a nonempty, closed, and convex subset of E. Let $T: C \rightarrow C$ be a Bregman relatively nonexpansive mapping. Then, $F(T)$ is closed and convex.

Lemma 3 (see [36]). Let $E$ be a reflexive Banach space and let $g: E \rightarrow \mathbb{R}$ be a convex, continuous, and strongly coercive function which is bounded on bounded subsets and uniformly convex on bounded subsets of $E$. Let $C$ be a nonempty, closed, and convex subset of $E$ and let $f$ be a bifunction from $C \times C$ to $\mathbb{R}$ satisfying (A1)-(A4). Let $r>0$ and $x \in E$. Then, there exists $z \in C$ such that $f(z, y)+1 / r\langle y-z, \nabla g z-\nabla g x\rangle \geq 0$, for all $y \in C$. Define a mapping $S_{r}: E \rightarrow C$ by $S_{r} x=\{z \in$ $C: f(z, y)+(1 / r)\langle y-z, \nabla g z-\nabla x\rangle$, for all $y \in C\}$. Then, the following conclusions hold:

(1) $S_{r}$ is single-valued;

(2) $S_{r}$ is a Bregman firmly nonexpansive-type mapping; that is, for all $x, y \in E$,

$$
\left\langle S_{r} x-S_{r} y, \nabla g S_{r} x-\nabla g S_{r} y\right\rangle \leq\left\langle S_{r} x-S_{r} y, \nabla x-\nabla g y\right\rangle ;
$$

(3) $F\left(S_{r}\right)=E P(f)$;

(4) $S_{r}$ is Bregman quasi-nonexpansive;

(5)

$$
D_{g}\left(q, S_{r} x\right)+D_{g}\left(S_{r} x, x\right) \leq \phi(q, x), \quad \forall q \in F\left(S_{r}\right)
$$

(6) $E P(f)$ is closed and convex.

\section{Main Results}

Theorem 4. Let $E$ be a reflexive Banach space and let $g: E \rightarrow$ $R$ be a strongly coercive Bregman function which is bounded on bounded subsets and uniformly convex and smooth on bounded subsets of E. Let $C$ be a nonempty, closed, and convex subset of $E$ and let $\Lambda$ be an index set. Let $\left\{s_{i}\right\}$ be a positive real number sequence. Let $f_{i}$ be a bifunction from $C \times C$ to $\mathbb{R}$ satisfying (A1)-(A4) and let $A_{i}: E \rightarrow E^{*}$ be a maximal monotone operator such that $\operatorname{Dom}\left(A_{i}\right) \subset C$ for every $i \in \Lambda$. Assume that the common solution set CSS $:=\cap_{i \in \Lambda} A_{i}^{-1}(0) \bigcap \cap_{i \in \Lambda} E F\left(f_{i}\right)$ is nonempty. Let $\left\{x_{n}\right\}$ be a sequence generated in the following manner:

$$
\begin{gathered}
x_{0} \in E \text { chosen arbitrarily, } \\
C_{(1, i)}=C, \quad C_{1}=\cap_{i \in \Lambda} C_{(1, i)}, \\
x_{1}=\operatorname{Proj}_{C_{1}}^{g} x_{0}, \\
y_{(n, i)}=\nabla g^{*}\left(\alpha_{(n, i)} \nabla g\left(x_{n}\right)+\left(1-\alpha_{(n, i)}\right) \nabla g J_{s_{i}}^{A_{i}} x_{n}\right),
\end{gathered}
$$


$u_{(n, i)} \in C$ such that

$$
\begin{array}{r}
f_{i}\left(u_{(n, i)}, y\right)+\frac{1}{r_{(n, i)}}\left\langle y-u_{(n, i)}, \nabla g\left(u_{(n, i)}\right)-\nabla g\left(y_{(n, i)}\right)\right\rangle \geq 0, \\
\forall y \in C, \\
C_{(n+1, i)}=\left\{z \in C_{(n, i)}: D_{g}\left(z, u_{(n, i)}\right) \leq D_{g}\left(z, x_{n}\right)\right\}, \\
C_{n+1}=\cap_{i \in \Lambda} C_{(n+1, i)}, \\
x_{n+1}=\operatorname{Proj}_{C_{n+1}}^{g} x_{1},
\end{array}
$$

where $\nabla g$ is the right-hand derivative of $g, J_{s_{i}}^{A_{i}}=(\nabla g+$ $\left.s_{i} A_{i}\right)^{-1} \nabla g,\left\{\alpha_{(n, i)}\right\}$ is a real sequence in $[0,1]$ such that $\liminf _{n \rightarrow \infty} \alpha_{(n, i)}\left(1-\alpha_{(n, i)}\right)>0$, and $\left\{r_{(n, i)}\right\}$ is a real sequence in $[a, \infty)$, where $a$ is some positive real number, for every $i \in \Lambda$. Then, the sequence $\left\{x_{n}\right\}$ converges strongly to Proj ${ }_{C S S}^{g} x_{1}$, where Proj $_{\text {CSS }}^{g}$ is the Bregman projection from E onto CSS.

Proof. In view of Lemma 3, we find that the common solution set CSS is closed and convex. Next, we prove that $C_{n}$ is closed and convex. It suffices to show that, for each fixed but arbitrary $i \in \Lambda, C_{(n, i)}$ is closed and convex. This can be proved by induction into $n$. It is obvious that $C_{(1, i)}=C$ is closed and convex. Assume that $C_{(k, i)}$ is closed and convex for some $k \geq 1$. For $z_{1}, z_{2} \in C_{(k+1, i)}$, we see that $z_{1}, z_{2} \in C_{(k, i)}$. It follows that $z=t z_{1}+(1-t) z_{2} \in C_{(k, i)}$, where $t \in(0,1)$. Notice that

$$
\begin{aligned}
& D_{g}\left(z_{1}, u_{(k, i)}\right) \leq D_{g}\left(z_{1}, x_{k}\right), \\
& D_{g}\left(z_{2}, u_{(k, i)}\right) \leq D_{g}\left(z_{2}, x_{k}\right) .
\end{aligned}
$$

The above inequalities are equivalent to

$$
\begin{aligned}
& \left\langle z_{1}, \nabla g(x)-\nabla g\left(u_{(k, i)}\right)\right\rangle \leq g\left(u_{(k, i)}\right)-g\left(x_{k}\right) \\
& -\left\langle u_{(k, i)}, \nabla g\left(u_{(k, i)}\right)\right\rangle+\langle x, \nabla g(x)\rangle, \\
& \left\langle z_{2}, \nabla g(x)-\nabla g\left(u_{(k, i)}\right)\right\rangle \leq g\left(u_{(k, i)}\right)-g\left(x_{k}\right) \\
& -\left\langle u_{(k, i)}, \nabla g\left(u_{(k, i)}\right)\right\rangle+\langle x, \nabla g(x)\rangle .
\end{aligned}
$$

Multiplying $t$ and $(1-t)$ on both sides of (21), respectively, yields that

$$
\begin{gathered}
\left\langle z, \nabla g(x)-\nabla g\left(u_{(k, i)}\right)\right\rangle \leq g\left(u_{(k, i)}\right)-g\left(x_{k}\right) \\
-\left\langle u_{(k, i)}, \nabla g\left(u_{(k, i)}\right)\right\rangle+\langle x, \nabla g(x)\rangle .
\end{gathered}
$$

That is,

$$
D_{g}\left(z, u_{(k, i)}\right) \leq D_{g}\left(z, x_{k}\right)
$$

where $z \in C_{(k, i)}$. This implies that $C_{(k+1, i)}$ is closed and convex. We conclude that $C_{(n, i)}$ is closed and convex. This in turn implies that $C_{n}=\cap_{i \in \Lambda} C_{(n, i)}$ is closed and convex. This implies that $\operatorname{Proj}_{C_{n+1}}^{g} x_{1}$ is well defined.
Next, we show that CSS $\subset C_{n}$. CSS $\subset C_{1}=C$ is clear. Suppose that CSS $\subset C_{(k, i)}$ for some positive integer $k$. For any $w \in \operatorname{CSS} \subset C_{(k, i)}$, we see that

$$
\begin{aligned}
D_{g}\left(w, u_{(k, i)}\right)= & D_{g}\left(w, S_{r_{(k, i)}} y_{(k, i)}\right) \\
\leq & D_{g}\left(w, y_{(k, i)}\right) \\
= & D_{g}\left(w, \nabla g^{*}\left(\alpha_{(k, i)} \nabla g\left(x_{k}\right)\right.\right. \\
& \left.\left.+\left(1-\alpha_{(k, i)}\right) \nabla g J_{s_{i}}^{A_{i}} x_{k}\right)\right) \\
= & V\left(w, \alpha_{(k, i)} \nabla g\left(x_{k}\right)+\left(1-\alpha_{(k, i)}\right) \nabla g\left(J_{s_{i}}^{A_{i}} x_{k}\right)\right) \\
= & g(w)-\alpha_{(k, i)}\left\langle w, \nabla g\left(x_{k}\right)\right\rangle-\left(1-\alpha_{(k, i)}\right) \\
& \times\left\langle w, \nabla g\left(J_{s_{i}}^{A_{i}} x_{k}\right)\right\rangle \\
& +g^{*}\left(\alpha_{(k, i)} \nabla g\left(x_{k}\right)+\left(1-\alpha_{(k, i)}\right) \nabla g\left(J_{s_{i}}^{A_{i}} x_{k}\right)\right) \\
\leq & \alpha_{(k, i)} V\left(w, \nabla g\left(x_{k}\right)\right) \\
& +\left(1-\alpha_{(k, i)}\right) V\left(w, \nabla g\left(J_{s_{i}}^{A_{i}} x_{k}\right)\right) \\
\leq & \alpha_{(k, i)} D_{g}\left(w, x_{k}\right)+\left(1-\alpha_{(k, i)}\right) D_{g}\left(w, J_{s_{i}}^{A_{i}} x_{k}\right) \\
\leq & D_{g}\left(w, x_{k}\right),
\end{aligned}
$$

which shows that $w \in C_{(k+1, i)}$. This implies that CSS $\subset C_{(n, i)}$. This yields that CSS $\subset \cap_{i \in \Lambda} C_{(n, i)}$. This completes the proof that CSS $\subset C_{n}$.

Next, we show that the sequence $\left\{x_{n}\right\}$ is bounded. It follows from (12) that

$$
\begin{aligned}
D_{g}\left(x_{n}, x_{1}\right) & \leq D_{g}\left(\operatorname{Proj}_{\mathrm{CSS}}^{g} x_{1}, x_{1}\right)-D_{g}\left(\operatorname{Proj}_{\mathrm{CSS}}^{g} x_{1}, x_{n}\right) \\
& \leq D_{g}\left(\operatorname{Proj}_{\mathrm{CSS}}^{g} x_{1}, x_{1}\right) .
\end{aligned}
$$

This implies that the sequence $\left\{D_{g}\left(x_{n}, x_{1}\right)\right\}$ is bounded. It follows that the sequence $\left\{x_{n}\right\}$ is also bounded. In view of the construction of the set $C_{n}$, we find that $C_{m} \subset C_{n}$ and $x_{m}=\operatorname{proj}_{C_{m}}^{g} x_{1} \in C_{m} \subset C_{n}$ for any positive integer $m \geq n$. It follows from (12) that

$$
\begin{aligned}
D_{g}\left(x_{m}, x_{n}\right) & =D_{g}\left(x_{m}, \operatorname{Proj}_{C_{n}}^{g} x_{1}\right) \\
& \leq D_{g}\left(x_{m}, x_{1}\right)-D_{g}\left(\operatorname{Proj}_{C_{n}}^{g} x_{1}, x_{1}\right) \\
& =D_{g}\left(x_{m}, x_{1}\right)-D_{g}\left(x_{n}, x_{1}\right) .
\end{aligned}
$$

It follows that

$$
D_{g}\left(x_{n}, x_{1}\right) \leq D_{g}\left(x_{n}, x_{1}\right)+D_{g}\left(x_{m}, x_{n}\right) \leq D_{g}\left(x_{m}, x_{1}\right) .
$$

This shows that the sequence $\left\{D_{g}\left(x_{n}, x_{1}\right)\right\}$ is nondecreasing. Hence, the limit $\lim _{n \rightarrow \infty} D_{g}\left(x_{n}, x_{1}\right)$ exists. In view of $x_{n}=$ $\operatorname{Proj}_{C_{n}}^{g} x_{1}$, we find that

$$
D_{g}\left(x_{n}, x_{1}\right) \leq D_{g}\left(x_{n+1}, x_{1}\right) \text {. }
$$


This concludes that $\lim _{n \rightarrow \infty} D_{g}\left(x_{n}, x_{1}\right)$ exists. Let $m, n \rightarrow$ $\infty$ in (26); we find that $D_{g}\left(x_{m}, x_{n}\right) \rightarrow 0$. In view of Lemma 1 , we obtain that $\left\|x_{m}-x_{n}\right\| \rightarrow 0$ as $m, n \rightarrow \infty$. This yields that $\left\{x_{n}\right\}$ is a Cauchy sequence. Since $C$ is closed and convex, we see that there exists an $\bar{x} \in C$; that is,

$$
\lim _{n \rightarrow \infty}\left\|x_{n}-\bar{x}\right\|=0
$$

Next, we show that $\bar{x} \in$ CSS. It follows from (26) that

$$
\lim _{n \rightarrow \infty} D_{g}\left(x_{n+1}, x_{n}\right)=0 \text {. }
$$

Therefore, we obtain that

$$
\lim _{n \rightarrow \infty}\left\|x_{n+1}-x_{n}\right\|=0
$$

Since $x_{n+1} \in C_{n+1}$, we find that

$$
D_{g}\left(x_{n+1}, u_{(n, i)}\right) \leq D_{g}\left(x_{n+1}, x_{n}\right) \text {. }
$$

In view of (30), we find that

$$
\lim _{n \rightarrow \infty} D_{g}\left(x_{n+1}, u_{(n, i)}\right)=0
$$

It follows that

$$
\lim _{n \rightarrow \infty}\left\|x_{n+1}-u_{(n, i)}\right\|=0 .
$$

Notice that

$$
\left\|x_{n}-u_{(n, i)}\right\| \leq\left\|x_{n}-x_{n+1}\right\|+\left\|x_{n+1}-u_{(n, i)}\right\| .
$$

It follows from (29) and (34) that $\lim _{n \rightarrow \infty}\left\|x_{n}-u_{(n, i)}\right\|=0$. In view of Lemma 1, we find that

$$
\lim _{n \rightarrow \infty} D_{g}\left(u_{(n, i)}, x_{n}\right)=0 \text {. }
$$

Since $\nabla g$ is uniformly norm-to-norm continuous on any bounded subset of $E$, we find that

$$
\lim _{n \rightarrow \infty}\left\|\nabla g\left(u_{(n, i)}\right)-\nabla g\left(x_{n}\right)\right\|=0
$$

Notice that

$$
\begin{aligned}
& \left|D_{g}\left(w, x_{n}\right)-D_{g}\left(w, u_{(n, i)}\right)\right| \\
& \quad=\left|D_{g}\left(u_{(n, i)}, x_{n}\right)+w-u_{(n, i)},\left\langle\nabla g\left(u_{(n, i)}\right)-\nabla g\left(x_{n}\right)\right\rangle\right| \\
& \quad \leq\left|D_{g}\left(u_{(n, i)}, x_{n}\right)\right|+\left\|w-u_{(n, i)}\right\|\left\|\nabla g\left(u_{(n, i)}\right)-\nabla g\left(x_{n}\right)\right\| .
\end{aligned}
$$

It follows from (36) and (37) that

$$
\lim _{n \rightarrow \infty}\left|D_{g}\left(w, x_{n}\right)-D_{g}\left(w, u_{(n, i)}\right)\right|=0
$$

In view of (14), we find that

$$
\begin{aligned}
& D_{g}\left(w, u_{(n, i)}\right)=D_{g}\left(w, S_{r_{(n, i)}} y_{(n, i)}\right) \\
& \leq D_{g}\left(w, y_{(n, i)}\right) \\
& =D_{g}\left(w, \nabla g^{*}\left(\alpha_{(n, i)} \nabla g\left(x_{n}\right)\right.\right. \\
& \left.\left.+\left(1-\alpha_{(n, i)}\right) \nabla g\left(J_{s_{i}}^{A_{i}} x_{n}\right)\right)\right) \\
& =V\left(w, \alpha_{(n, i)} \nabla g\left(x_{n}\right)+\left(1-\alpha_{(n, i)}\right) \nabla g\left(J_{s_{i}}^{A_{i}} x_{n}\right)\right) \\
& =g(w)-\alpha_{(n, i)}\left\langle w, \nabla g\left(x_{n}\right)\right\rangle-\left(1-\alpha_{(n, i)}\right) \\
& \times\left\langle w, \nabla g\left(J_{s_{i}}^{A_{i}} x_{n}\right)\right\rangle \\
& +g^{*}\left(\alpha_{(n, i)} \nabla g\left(x_{n}\right)+\left(1-\alpha_{(n, i)}\right) \nabla g\left(J_{s_{i}}^{A_{i}} x_{n}\right)\right) \\
& \leq \alpha_{(n, i)} V\left(w, \nabla g\left(x_{n}\right)\right) \\
& +\left(1-\alpha_{(n, i)}\right) V\left(w, \nabla g\left(J_{s_{i}}^{A_{i}} x_{n}\right)\right) \\
& -\rho\left(\left\|\nabla g\left(J_{s_{i}}^{A_{i}} x_{n}\right)-\nabla g\left(x_{n}\right)\right\|\right) \\
& \leq \alpha_{(n, i)} D_{g}\left(w, x_{n}\right)+\left(1-\alpha_{(n, i)}\right) D_{g}\left(w, J_{s_{i}}^{A_{i}} x_{n}\right) \\
& -\alpha_{(n, i)}\left(1-\alpha_{(n, i)}\right) \\
& \times \rho\left(\left\|\nabla g\left(J_{s_{i}}^{A_{i}} x_{n}\right)-\nabla g\left(x_{n}\right)\right\|\right) \\
& \leq D_{g}\left(w, x_{n}\right)-\alpha_{(n, i)}\left(1-\alpha_{(n, i)}\right) \\
& \times \rho\left(\left\|\nabla g\left(J_{s_{i}}^{A_{i}} x_{n}\right)-\nabla g\left(x_{n}\right)\right\|\right),
\end{aligned}
$$

where $\rho$ is the gauge of the uniform convexity of $g^{*}$. It follows that

$$
\begin{aligned}
& \alpha_{(n, i)}\left(1-\alpha_{(n, i)}\right) \rho\left(\left\|\nabla g\left(J_{s_{i}}^{A_{i}} x_{n}\right)-\nabla g\left(x_{n}\right)\right\|\right) \\
& \leq D_{g}\left(w, x_{n}\right)-D_{g}\left(w, u_{(n, i)}\right) .
\end{aligned}
$$

In view of the restrictions on the sequence $\left\{\alpha_{(n, i)}\right\}$, we find from (39) that $\lim _{n \rightarrow \infty}\left\|\nabla g\left(x_{n}\right)-\nabla g\left(J_{s_{i}}^{A_{i}} x_{n}\right)\right\|=0$. Since $\nabla g^{*}$ is uniformly norm-to-norm continuous on bounded subsets of $E^{*}$, we obtain that

$$
\lim _{n \rightarrow \infty}\left\|x_{n}-J_{s_{i}}^{A_{i}} x_{n}\right\|=0 .
$$

Since $J_{s_{i}}^{A_{i}}$ is closed Bregman quasi-nonexpansive, we find that $J_{s_{i}}^{A_{i}} \bar{x}=\bar{x}$. This proves that $\bar{x} \in \cap_{i \in \Lambda} A_{i}^{-1}(0)$.

Next, we show that $\bar{x} \in \cap_{i \in \Lambda} \operatorname{EF}\left(f_{i}\right)$. Notice that

$$
\begin{aligned}
D_{g}\left(u_{(n, i)}, y_{(n, i)}\right) & \leq D_{g}\left(w, y_{(n, i)}\right)-D_{g}\left(w, u_{(n, i)}\right) \\
& \leq D_{g}\left(w, x_{n}\right)-D_{g}\left(w, u_{(n, i)}\right) .
\end{aligned}
$$

It follows from (39) that

$$
\lim _{n \rightarrow \infty} D_{g}\left(u_{(n, i)}, y_{(n, i)}\right)=0 .
$$


Therefore, we find that $\lim _{n \rightarrow \infty}\left\|u_{(n, i)}-y_{(n, i)}\right\|=0$. Since $\nabla g$ is uniformly norm-to-norm continuous on any bounded sets, we have $\lim _{n \rightarrow \infty}\left\|\nabla g\left(u_{(n, i)}\right)-\nabla\left(y_{(n, i)}\right)\right\|=0$. From the assumption $r_{(n, i)} \geq a$, we see that

$$
\lim _{n \rightarrow \infty} \frac{\left\|\nabla g\left(u_{(n, i)}\right)-\nabla g\left(y_{(n, i)}\right)\right\|}{r_{(n, i)}}=0 .
$$

In view of $u_{(n, i)}=S_{r_{(n, i)}} y_{(n, i)}$, we see that

$$
\begin{array}{r}
f_{i}\left(u_{(n, i)}, y\right)+\frac{1}{r_{(n, i)}}\left\langle y-u_{(n, i)}, \nabla g\left(u_{(n, i)}\right)-\nabla g\left(y_{(n, i)}\right)\right\rangle \geq 0, \\
\forall y \in C .
\end{array}
$$

It follows from (A2) that

$$
\begin{aligned}
\| y & -u_{(n, i)} \| \frac{\left\|\nabla g\left(u_{(n, i)}\right)-\nabla g\left(y_{(n, i)}\right)\right\|}{r_{n, i}} \\
& \geq \frac{1}{r_{(n, i)}}\left\langle y-u_{(n, i)}, \nabla g\left(u_{(n, i)}\right)-\nabla g\left(y_{(n, i)}\right)\right\rangle \\
& \geq f_{i}\left(y, u_{(n, i)}\right), \quad \forall y \in C .
\end{aligned}
$$

In view of (A4), we find from (45) that

$$
f_{i}(y, \bar{x}) \leq 0, \quad \forall y \in C .
$$

For $0<t_{i}<1$ and $y \in C$, define $y_{(t, i)}=t_{i} y+\left(1-t_{i}\right) \bar{x}$. It follows that $y_{(t, i)} \in C$, which yields that $f\left(y_{(t, i)}, \bar{x}\right) \leq 0$. It follows from (A1) and (A4) that

$$
\begin{aligned}
0 & =f\left(y_{(t, i)}, y_{(t, i)}\right) \\
& \leq t_{i} f\left(y_{(t, i)}, y\right)+\left(1-t_{i}\right) f\left(y_{(t, i)}, \bar{x}\right) \\
& \leq t_{i} f\left(y_{(t, i)}, y\right) .
\end{aligned}
$$

That is,

$$
f\left(y_{(t, i)}, y\right) \geq 0 .
$$

Letting $t_{i} \downarrow 0$, we obtain from (A3) that $f_{i}(\bar{x}, y) \geq 0$, for all $y \in C$. This implies that $\bar{x} \in \operatorname{EP}\left(f_{i}\right)$ for every $i \in \Lambda$. This shows that $\bar{x} \in \mathrm{CSS}$.

Finally, we prove that $\bar{x}=\operatorname{Proj}_{\mathrm{CSS}}^{g} x_{1}$. In view of $x_{n}=$ $\operatorname{Proj}_{C_{n}}^{g} x_{1}$, we conclude that

$$
\left\langle z-x_{n}, \nabla g\left(x_{n}\right)-\nabla g\left(x_{1}\right)\right\rangle, \quad \forall z \in C_{n} .
$$

Since $\operatorname{CSC} \subset C_{n}$, we arrive at

$$
\left\langle w-x_{n}, \nabla g\left(x_{n}\right)-\nabla g\left(x_{1}\right)\right\rangle, \quad \forall w \in \text { CSC. }
$$

Letting $n \rightarrow \infty$ in the above inequality, we see that

$$
\left\langle w-\bar{x}, \nabla g(\bar{x})-\nabla g\left(x_{1}\right)\right\rangle \geq 0, \quad \forall w \in \text { CSS. }
$$

This yields that $\bar{x}=\operatorname{Proj}_{\mathrm{CSS}}^{g} x_{1}$. This completes the proof. result.

If the index set is singleton, then we have the following
Corollary 5. Let $E$ be a reflexive Banach space and let $g: E \rightarrow$ $R$ be a strongly coercive Bregman function which is bounded on bounded subsets and uniformly convex and smooth on bounded subsets of E. Let $C$ be a nonempty, closed, and convex subset of $E$ and let $s$ be a positive real number. Let $f$ be a bifunction from $C \times C$ to $\mathbb{R}$ satisfying (A1)-(A4) and let $A: E \rightarrow E^{*}$ be a maximal monotone operator such that $\operatorname{Dom}(A) \subset C$. Assume that the common solution set CSS $:=A^{-1}(0) \bigcap E F(f)$ is nonempty. Let $\left\{x_{n}\right\}$ be a sequence generated in the following manner:

$$
\begin{gathered}
x_{0} \in E \text { chosen arbitrarily, } \\
C_{1}=C, \\
x_{1}=\operatorname{Proj}_{C_{1}}^{g} x_{0}, \\
y_{n}=\nabla g^{*}\left(\alpha_{n} \nabla g\left(x_{n}\right)+\left(1-\alpha_{n}\right) \nabla g\left(J_{s}^{A} x_{n}\right)\right), \\
u_{n} \in C \text { such that } \\
f\left(u_{n}, y\right)+\frac{1}{r_{n}}\left\langle y-u_{n}, \nabla g\left(u_{n}\right)-\nabla g\left(y_{n}\right)\right\rangle \geq 0, \\
C_{n+1}=\left\{z \in C_{n}: D_{g}\left(z, u_{n}\right) \leq D_{g}\left(z, x_{n}\right)\right\}, \\
x_{n+1}=\operatorname{Proj}_{C_{n+1}}^{g} x_{1},
\end{gathered}
$$

where $\nabla g$ is the right-hand derivative of $g . J_{s}^{A}=$ $(\nabla g+s A)^{-1} \nabla g, \quad\left\{\alpha_{n}\right\}$ is a real sequence in $[0,1]$ such that $\liminf _{n \rightarrow \infty} \alpha_{n}\left(1-\alpha_{n}\right)>0$, and $\left\{r_{n}\right\}$ is a real sequence in $[a, \infty)$, where $a$ is some positive real number. Then, the sequence $\left\{x_{n}\right\}$ converges strongly to $\operatorname{Proj}_{\text {CSS }}^{g} x_{1}$, where $\operatorname{Proj}_{\text {CSS }}^{g}$ is the Bregman projection from $E$ onto CSS.

Remark 6. Corollary 5 improves Theorem 4 of Qin et al. [24] in the following aspects: (1) the framework of spaces is relaxed; (2) a more general notion of Bregman function is considered instead of the generalized duality mapping; (3) a more general notion of Bregman projection is considered instead of the generalized projection operator; (4) families of operators extend from a finite family to an infinite family.

Finally, we give some results in the framework of Hilbert spaces.

Theorem 7. Let $E$ be a Hilbert space and let $C$ be a nonempty, closed, and convex subset of E. Let $\Lambda$ be an index set. Let $\left\{s_{i}\right\}$ be a positive real number sequence. Let $f_{i}$ be a bifunction from $C \times C$ to $\mathbb{R}$ satisfying (A1)-(A4) and let $A_{i}: E \rightarrow E$ be a maximal monotone operator such that $\operatorname{Dom}\left(A_{i}\right) \subset C$ for every $i \in \Lambda$. Assume that the common solution set CSS := $\cap_{i \in \Lambda} A_{i}^{-1}(0) \bigcap \cap_{i \in \Lambda} E F\left(f_{i}\right)$ is nonempty. Let $\left\{x_{n}\right\}$ be a sequence generated in the following manner:

$x_{0} \in E$ chosen arbitrarily,

$$
\begin{gathered}
C_{(1, i)}=C, \\
C_{1}=\cap_{i \in \Lambda} C_{(1, i)}, \\
x_{1}=\operatorname{Proj}_{C_{1}} x_{0}, \\
y_{(n, i)}=\alpha_{(n, i)} x_{n}+\left(1-\alpha_{(n, i)}\right) J_{s_{i}}^{A_{i}} x_{n},
\end{gathered}
$$




$$
\begin{gathered}
u_{(n, i)} \in C \text { such that } \\
f_{i}\left(u_{(n, i)}, y\right)+\frac{1}{r_{(n, i)}}\left\langle y-u_{(n, i)}, u_{(n, i)}-y_{(n, i)}\right\rangle \geq 0, \\
\forall y \in C, \\
C_{(n+1, i)}=\left\{z \in C_{(n, i)}:\left\|z-u_{(n, i)}\right\|^{2} \leq\left\|z-x_{n}\right\|^{2}\right\}, \\
C_{n+1}=\cap_{i \in \Lambda} C_{(n+1, i)}, \\
x_{n+1}=\operatorname{Proj}_{C_{n+1}} x_{1},
\end{gathered}
$$

where $J_{s_{i}}^{A_{i}}=\left(I+s_{i} A_{i}\right)^{-1},\left\{\alpha_{(n, i)}\right\}$ is a real sequence in $[0,1]$ such that $\liminf _{n \rightarrow \infty} \alpha_{(n, i)}\left(1-\alpha_{(n, i)}\right)>0$, and $\left\{r_{(n, i)}\right\}$ is a real sequence in $[a, \infty)$, where $a$ is some positive real number, for every $i \in \Lambda$. Then, the sequence $\left\{x_{n}\right\}$ converges strongly to $\operatorname{Proj}_{\mathrm{CSS}} x_{1}$, where Proj ${ }_{\text {CSS }}$ is the metric projection from $E$ onto CSS.

Proof. Putting $g(x)=(1 / 2)\|x\|^{2}$, we find that $\nabla g(x)=x$ for all $x \in E$. It follows that $D_{g}(x, y)=\|x-y\|^{2}$ for all $x, y \in$ $E$. From Theorem 4, we can immediately obtain the desired conclusion. result.

If the index set is singleton, then we have the following

Corollary 8. Let $E$ be a Hilbert space and let $C$ be a nonempty, closed, and convex subset of E. Let $s$ be a positive real number. Let $f$ be a bifunction from $C \times C$ to $\mathbb{R}$ satisfying (A1)-(A4) and let $A: E \rightarrow E$ be a maximal monotone operator such that $\operatorname{Dom}(A) \subset C$. Assume that the common solution set CSS := $A^{-1}(0) \bigcap E F(f)$ is nonempty. Let $\left\{x_{n}\right\}$ be a sequence generated in the following manner:

$$
\begin{gathered}
x_{0} \in E \text { chosen arbitrarily, } \\
C_{1}=C, \\
x_{1}=\operatorname{Proj}_{C_{1}} x_{0}, \\
y_{n}=\alpha_{n} x_{n}+\left(1-\alpha_{n}\right) J_{s}^{A} x_{n},
\end{gathered}
$$

$u_{n} \in C$ such that

$$
\begin{gathered}
f\left(u_{n}, y\right)+\frac{1}{r_{n}}\left\langle y-u_{n}, u_{n}-y_{n}\right\rangle \geq 0, \quad \forall y \in C, \\
C_{n+1}=\left\{z \in C_{n}:\left\|z-u_{n}\right\|^{2} \leq\left\|z-x_{n}\right\|^{2}\right\}, \\
x_{n+1}=\operatorname{Proj}_{C_{n+1}} x_{1},
\end{gathered}
$$

where $J_{s}^{A}=(I+s A)^{-1},\left\{\alpha_{n}\right\}$ is a real sequence in $[0,1]$ such that $\liminf _{n \rightarrow \infty} \alpha_{n}\left(1-\alpha_{n}\right)>0$ and $\left\{r_{n}\right\}$ is a real sequence in $[a, \infty)$, where $a$ is some positive real number. Then, the sequence $\left\{x_{n}\right\}$ converges strongly to $\operatorname{Proj}_{\mathrm{CSS}} x_{1}$, where $\operatorname{Proj}_{\mathrm{CSS}}$ is the metric projection from $E$ onto $C S S$.

\section{References}

[1] H. Iiduka, "Fixed point optimization algorithm and its application to network bandwidth allocation," Journal of Computational and Applied Mathematics, vol. 236, no. 7, pp. 1733-1742, 2012.

[2] G. Wang and S. Sun, "Hybrid projection algorithms for fixed point and equilibrium problems in a Banach space," Advances in Fixed Point Theory, vol. 3, pp. 578-594, 2013.

[3] S. Y. Cho, W. Li, and S. M. Kang, "Convergence analysis of an iterative algorithm for monotone operators," Journal of Inequalities and Applications, vol. 2013, article 199, 2013.

[4] J. K. Kim, "Strong convergence theorems by hybrid projection methods for equilibrium problems and fixed point problems of the asymptotically quasi- $\phi$-nonexpansive mappings," Fixed Point Theory and Applications, vol. 2011, article 10, 2011.

[5] S. Park, "A review of the KKM theory on $\phi_{A}$-space or GFCspaces," Advances in Fixed Point Theory, vol. 3, pp. 355-382, 2013.

[6] S. Y. Cho and S. M. Kang, "Approximation of fixed points of pseudocontraction semigroups based on a viscosity iterative process," Applied Mathematics Letters, vol. 24, no. 2, pp. 224228, 2011.

[7] S. Lv, "Strong convergence of a general iterative algorithm in Hilbert spaces," Journal of Inequalities and Applications, vol. 2013, article 19, 2013.

[8] R. H. He, "Coincidence theorem and existence theorems of solutions for a system of Ky Fan type minimax inequalities in FC-spaces," Advances in Fixed Point Theory, vol. 2, pp. 47-57, 2012.

[9] S. Y. Cho, X. Qin, and S. M. Kang, "Iterative processes for common fixed points of two different families of mappings with applications," Journal of Global Optimization, vol. 57, no. 4, pp. 1429-1446, 2013.

[10] S. Y. Cho and S. M. Kang, "Approximation of common solutions of variational inequalities via strict pseudocontractions," Acta Mathematica Scientia, vol. 32, no. 4, pp. 1607-1618, 2012.

[11] B. C. Dhage and N. S. Jadhav, "Differential inequalities and comparison theorems for first order hybrid integro-differential equations," Advances in Inequalities and Applications, vol. 2, no. 1, pp. 61-80, 2013.

[12] L. J. Lin, S. F. Cheng, X. Y. Liu, and Q. H. Ansari, "On the constrained equilibrium problems with finite families of players," Nonlinear Analysis: Theory, Methods and Applications, vol. 54, no. 3, pp. 525-543, 2003.

[13] X. Qin, S. Y. Cho, and S. M. Kang, "Iterative algorithms for variational inequality and equilibrium problems with applications," Journal of Global Optimization, vol. 48, no. 3, pp. 423-445, 2010.

[14] C. Wu and G. Wang, "Hybrid projection algorithms for asymptotically quasi- $\phi$-nonexpansive mappings," Communications in Optimization Theory, vol. 2013, article 2, 2013.

[15] X. Yang, "Iterative sequences with errors for generalized contractive mappings in metric spaces," Engineering Mathematics Letters, vol. 2, pp. 24-33, 2013.

[16] H. Zhang, "A general iterative algorithm for equilibrium problems and fixed point problems in Hilbert spaces," Fixed Point Theory and Applications, vol. 2013, article 1, 2013.

[17] A. Y. Al-Bayati and R. Z. Al-Kawaz, "A new hybrid WC-FR conjugate gradient-algorithm with modified secant condition for unconstrained optimization," Journal of Mathematics and Computer Science, vol. 2, pp. 937-966, 2012. 
[18] Z. M. Wang and W. Lou, "A new iterative algorithm of common solutions to quasi-variational inclusionand fixed point problems," Journal of Mathematics and Computer Science, vol. 3, pp. 57-72, 2013.

[19] C. Byrne, "A unified treatment of some iterative algorithms in signal processing and image reconstruction," Inverse Problems, vol. 20, no. 1, pp. 103-120, 2004.

[20] B. O. Osu and O. U. Solomon, "A stochastic algorithm for the valuation of financial derivatives using the hyperbolic distributional variates," Mathematical Finance Letters, vol. 1, pp. 43-56, 2012.

[21] Y. Tanaka, "A constructive version of Ky Fan's coincidence theorem," Journal of Mathematical and Computational Science, vol. 2, pp. 926-936, 2012.

[22] X. Qin, S. Y. Cho, and S. M. Kang, "An extragradient-type method for generalized equilibrium problems involving strictly pseudocontractive mappings," Journal of Global Optimization, vol. 49, no. 4, pp. 679-693, 2011.

[23] G. Lopez, V. Martin, and H. K. Xu, "Perturbation techniques for nonexpansive mappings with applications," Nonlinear Analysis: Real World Applications, vol. 10, no. 4, pp. 2369-2383, 2009.

[24] X. Qin, Y. J. Cho, and S. M. Kang, "Convergence theorems of common elements for equilibrium problems and fixed point problems in Banach spaces," Journal of Computational and Applied Mathematics, vol. 225, no. 1, pp. 20-30, 2009.

[25] S. S. Chang, J. C. Yao, J. K. Kim, and L. Yang, "Iterative approximation to convex feasibility problems in Banach space," Fixed Point Theory and Applications, vol. 2007, Article ID 46797, 2007.

[26] H. H. Bauschke and J. M. Borwein, "On projection algorithms for solving convex feasibility problems," SIAM Review, vol. 38, no. 3, pp. 367-426, 1996.

[27] A. S. Saluja and P. K. Jhade, "Common fixed points of two generalized quasi-nonexpansive mapping by a new three-step iteration scheme," Advances in Fixed Point Theory, vol. 3, pp. 263-272, 2013.

[28] X. Qin, Y. J. Cho, S. M. Kang, and H. Zhou, "Convergence theorems of common fixed points for a family of Lipschitz quasipseudocontractions," Nonlinear Analysis: Theory, Methods and Applications, vol. 71, no. 1-2, pp. 685-690, 2009.

[29] F. E. Browder, "Nonlinear mappings of nonexpansive and accretive type in Banach spaces," Bulletin of the American Mathematical Society, vol. 73, pp. 875-882, 1967.

[30] Y. Haugazeau, Sur les inéquations variationnelles et la minimisation de fonctionnelles convexes [Ph.D. thesis], Université de Paris, Paris, France, 1968.

[31] C. Zalinescu, Convex Analysis in General Vextor Spaces, World Scientific, River Edge, NJ, USA, 2002.

[32] D. Butnariu and A. N. Iusem, Totally Convex Functions for Fixed Points Computation and in Finite Dimensional Optimization,, Kluwer Academic, Dordrecht, The Netherlands, 2000.

[33] R. T. Rockafellar, "On the maximality of sums of nonlinear monotone operators," Transactions of the American Mathematical Society, vol. 149, pp. 75-88, 1970.

[34] E. Naraghirad and J. C. Yao, "Bregman weak relatively nonexpansive mappings in Banach spaces," Fixed Point Theory and Applications, vol. 2013, article 141, 2013.

[35] S. Reich and S. Sabach, "Existence and approximation of fixed points of Bregman firmly nonexpansive mappings in reflexive Banach spaces," in Fixed-Point Algorithms for Inverse Problems in Science and Engineering, vol. 49 of Springer Optimization and Its Applications, pp. 301-316, Springer, New York, NY, USA, 2011.
[36] S. Reich and S. Sabach, "Two strong convergence theorems for Bregman strongly nonexpansive operators in reflexive Banach spaces," Nonlinear Analysis: Theory, Methods and Applications, vol. 73, no. 1, pp. 122-135, 2010. 


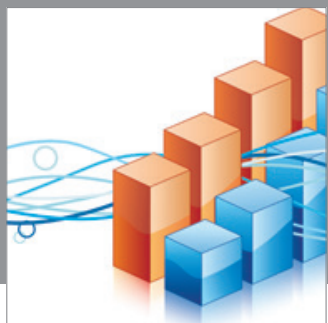

Advances in

Operations Research

mansans

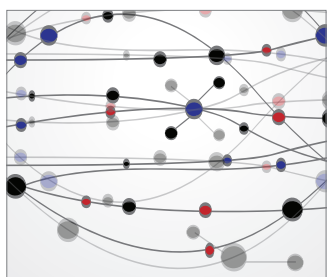

The Scientific World Journal
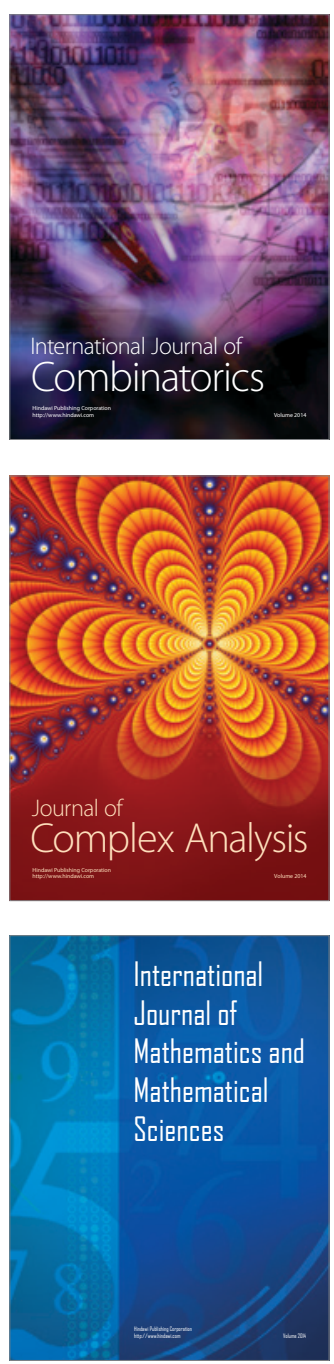
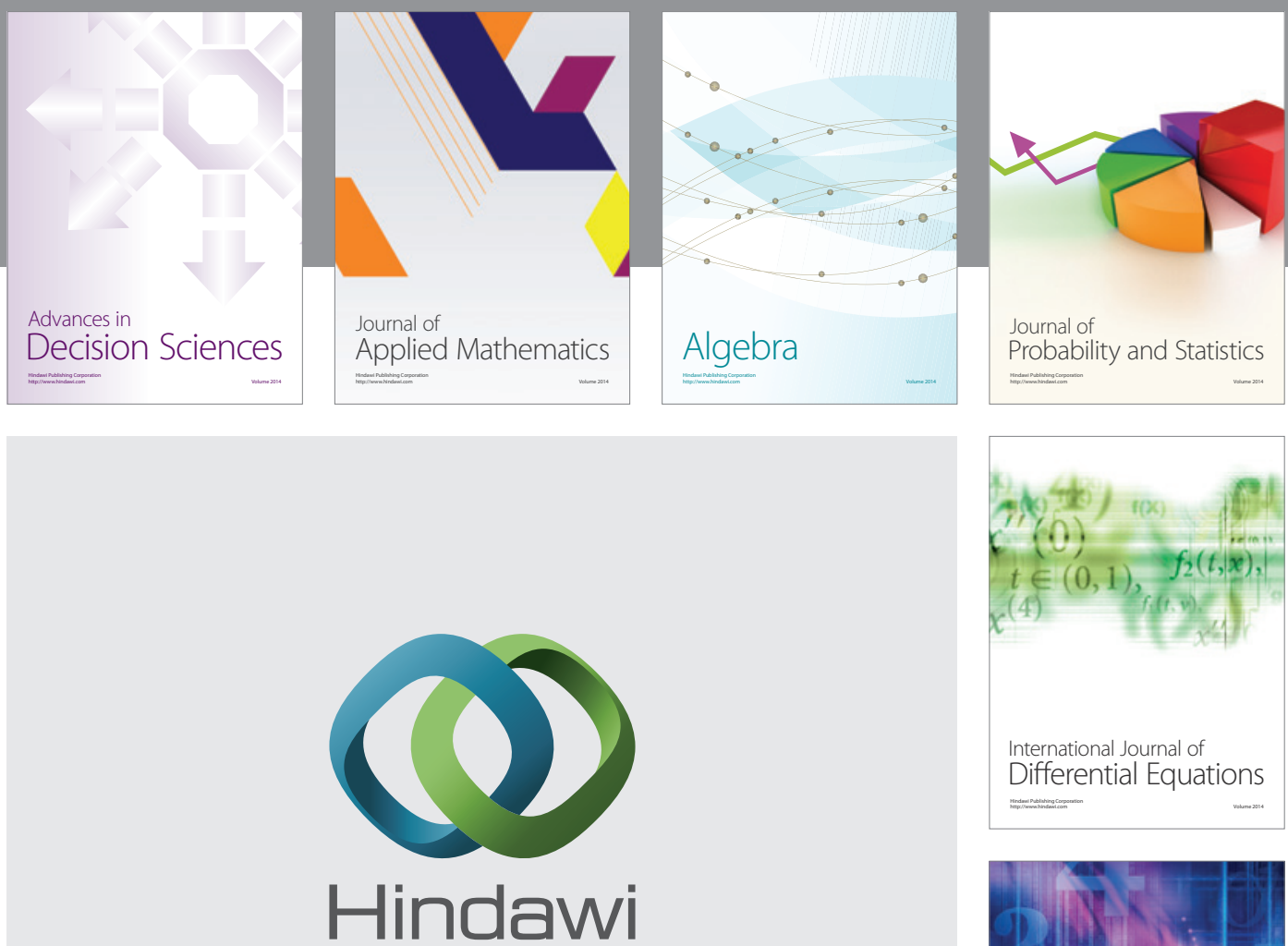

Submit your manuscripts at http://www.hindawi.com
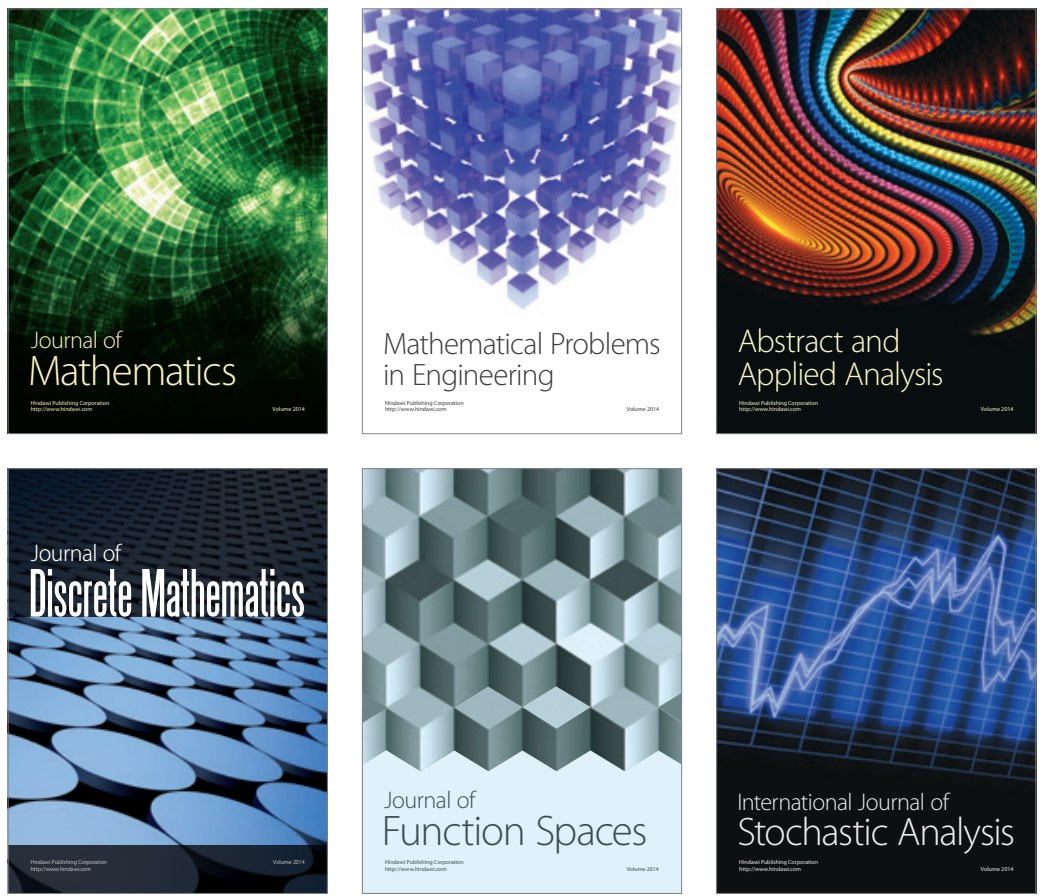

Journal of

Function Spaces

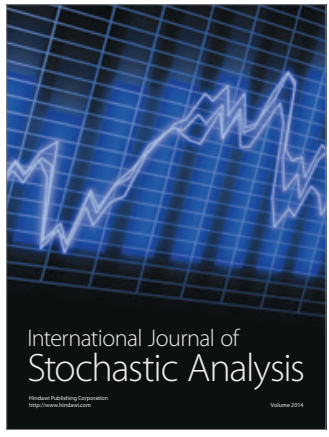

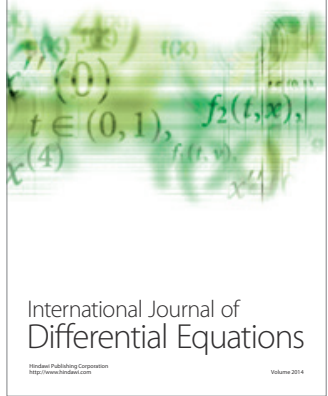
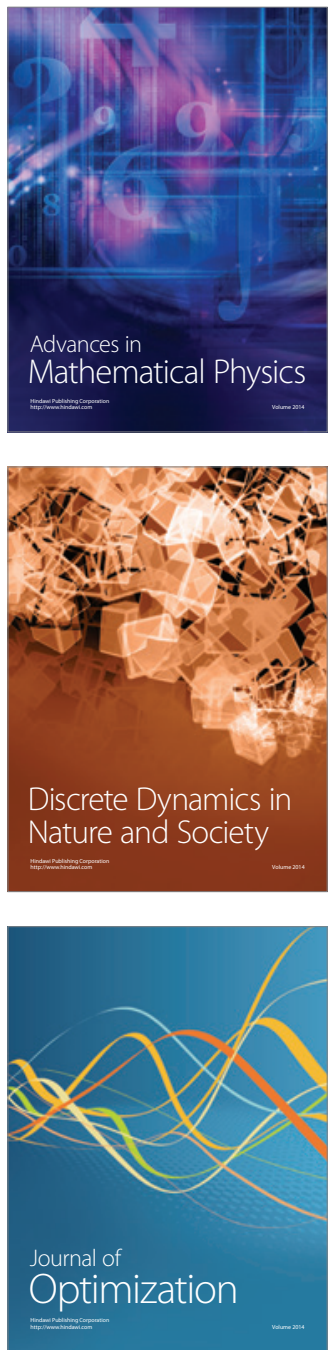